\title{
On the Problem of Eigenschaften in Quantum and Classical Mechanics
}

\author{
Daniel Sepunaru \\ RCQCE-Research Center for Quantum Communication, Holon Academic Institute of Technology, Holon, Israel \\ Email: danielsepunaru@walla.co.il
}

How to cite this paper: Sepunaru, D. (2018) On the Problem of Eigenschaften in Quantum and Classical Mechanics. Journal of Modern Physics, 9, 2378-2390. https://doi.org/10.4236/jmp.2018.914152

Received: October 3, 2018

Accepted: December 2, 2018

Published: December 5, 2018

Copyright (c) 2018 by author and Scientific Research Publishing Inc. This work is licensed under the Creative Commons Attribution International License (CC BY 4.0).

http://creativecommons.org/licenses/by/4.0/

\begin{abstract}
We argue that in contrast to the classical physics, measurements in quantum mechanics should provide simultaneous information about all relevant relative amplitudes (pure states and the transitions between them) and all relevant relative phases. Simultaneity is needed since measurement changes the state of the system (in both quantum and in classical physics). We call that measurement procedure holographic detection. Mathematically, it is described by a set of mutually commuting selfadjoint operators that are similar and closely related to projections. We present explicit examples and discuss general features of the corresponding experimental setup which we identify as the quantum reference frame.
\end{abstract}

\section{Keywords}

Hilbert Spaces, Holographic Detection, Quantum Reference Frames

\section{Introduction}

Debates about the connection between hidden laws of nature and our ability to extract the information necessary to formulate these laws have a long history, perhaps as long as study of physics itself. This paper, while not related to the philosophical or metaphysical aspects of those discussions, puts forth certain point of view without intention to defend it or to convince the reader that it is only possible approach. We simply present how the process of knowledge acquisition is realized within that approach. We explore the analogy to the structure of field theories (classical electrodynamics, general relativity and non-relativistic quantum mechanics) and make a distinction between unobservable kinematical quantities which characterize a physical system and the measurable variables which define its dynamics. Since the main distinction between classical and 
quantum physics is the presence of new kinematic quantities-phases-we need to know how to measure the corresponding phase differences. We demonstrate that this measurement may be obtained by using a special experimental arrangement that we call quantum reference frames. This allows for communicating the required hidden unobservable information to the instruments of the observer. This simultaneously explains why the elementary unit of communication is given in terms of an indivisible bit.

The notion of the eigenschaften operator was first introduced by J. von Neumann [1] as a necessary ingredient of his theory of measurements. He suggested assigning that role to projection operators which define not only the space of quantum mechanical states but also the structure of that space and its complete, orthonormal basis. In our model, it is logically consistent to use eigenschaften operators that closely relate to projection operators but act on the whole space without distortion; that is, eigenschaften operators that are unitary.

The main feature of the measurement process is that measurement devices are macroscopic, obeying the laws of classical physics, whereas the systems being tested belong to the microscopic world and behave quantum mechanically. Indeed, the measurement setup should assure that the results obtained represent objective properties of the physical system being investigated and not the subjective imagination of the observer. Using classical physics, we complete that task by introducing reference frames such that the location of the detector defines both the frame's origin and the set of auxiliary macroscopic devices. This allows for establishment of a connection between frames that are separated by a finite space-time interval (comparison of the empirical data obtained must always be performed by the same observer). Similarly, in order to measure the relevant quantum dynamical variable a set of auxiliary macroscopic devices should be included in the classical setup to produce the necessary beam-splitting. Then the required phase differences can be measured in the usual way. This setup and recording procedure may be viewed as general holographic detection.

The organization of this paper is as follows:

Section 2 presents a discussion of the relevant kinematics of the quantum theory. Section 3 introduces the unitary, self-adjoint operators which we identify as adequate eigenschaften operators.

Section 4 discusses the quantum frames of reference making a close analogy to the inertial frames of classical physics.

\section{The Kinematics of Quantum Mechanical Theory}

We restrict ourselves to discussion of single particle states, avoiding complications introduced by special relativity. We use an orthodox kinematic approach based on the mathematical framework of Hilbert metric spaces. That means that we assume that there exists at least one self-adjoin operator that generates this space. That operator is supposed to describe the dynamics of a single particle that is completely isolated from the external world. All measurable quantities are 
also described by self-adjoint operators. In particular, projection operators, density matrices, etc. are treated as special kinds of observables, whereas the fundamental quantity associated with the state of the physical system is treated as a wave function [2]. In contrast to operators that are geometric transformations of the given vector space, wave functions are vectors that form that space and are both unobservable and incapable of being measured directly, at least in principle.

The transition from the sterile situation of a single isolated particle to the real-life physical system is achieved through introduction of the local interactions of the test particle with the fields generated by the rest of the external world. These interactions are introduced using the principle of local gauge invariance. The required complexity emerges from the statistical nature of the environment. This approach is identical to the conventional one that has long been established in the development of classical physics over the centuries except that the definition of (fundamental) interactions is now connected to the new physics, since we are dealing with matter waves.

The fundamental property of the quantum mechanical states is as expressed in terms of the linear superposition principle is:

If $\left|\Psi_{1}\right\rangle$ and $\left|\Psi_{2}\right\rangle$ are two different states of the system, then

$$
|\Psi\rangle=a\left|\Psi_{1}\right\rangle+b\left|\Psi_{2}\right\rangle
$$

is also a state of the system. Equivalently, we may write:

$$
|\Psi\rangle=a\left(\begin{array}{c}
\left|\Psi_{1}\right\rangle \\
0
\end{array}\right)+b\left(\begin{array}{c}
0 \\
\left|\Psi_{2}\right\rangle
\end{array}\right)
$$

or

$$
|\Psi\rangle=\left(\begin{array}{l}
a\left|\Psi_{1}\right\rangle \\
b\left|\Psi_{2}\right\rangle
\end{array}\right) ;\left\langle\Psi_{1} \mid \Psi_{1}\right\rangle=\left\langle\Psi_{2} \mid \Psi_{2}\right\rangle=1 ;\left\langle\Psi_{1} \mid \Psi_{2}\right\rangle=0
$$

However, that seemingly innocent-looking mathematical expressions leads to a dramatic change in the physics of the described system, since the presence of the second orthogonal component is the necessary and sufficient condition that now the above function describes the extended object:

Theorem [3]: if $\hat{A}^{+}=\hat{A}$ and $\left\langle\Psi_{1} \mid \Psi_{2}\right\rangle=0 ;\left\langle\Psi_{1} \mid \Psi_{1}\right\rangle=\left\langle\Psi_{2} \mid \Psi_{2}\right\rangle=1$;

Then

$$
\begin{gathered}
\hat{A}\left|\Psi_{1}\right\rangle=a\left|\Psi_{1}\right\rangle+b\left|\Psi_{2}\right\rangle \\
a=\left\langle\Psi_{1}|\hat{A}| \Psi_{1}\right\rangle=\langle\hat{A}\rangle \equiv \bar{A} \\
|b|^{2}=b b^{*}=\left\langle\Psi_{1}\left|\left(\hat{A}^{2}-a^{2}\right)\right| \Psi_{1}\right\rangle \equiv(\Delta A)^{2}
\end{gathered}
$$

can be decomposed.

Proof:

$$
\begin{gathered}
\left\langle\Psi_{1}\left|\left(\hat{A}^{2}-a^{2}\right)\right| \Psi_{1}\right\rangle=\left\langle\Psi_{1}\left|\hat{A}^{2}\right| \Psi_{1}\right\rangle-a^{2}= \\
\left(a \left\langle\Psi_{1}\left|+b^{*}\left\langle\Psi_{2}\right|\right)\left(a\left|\Psi_{1}\right\rangle+b\left|\Psi_{2}\right\rangle\right)-a^{2}=b b^{*}\right.\right.
\end{gathered}
$$


Therefore, what we need to reconstruct in the properly performed quantum mechanical measurement is a picture. Since equations of motion are intrinsically complex, the quantum mechanical system must be described by a two-component state function at least, due to the Euler relation:

$$
\exp (i \varphi)=\cos \varphi+i \sin \varphi .
$$

In contrast to classical physics, quantum mechanics is the physics of extended objects; it is the theory of matter fields. Now, due to D. Hilbert's spectral decomposition theorem [4], any $\hat{A}$, such that $\hat{A}=\hat{A}^{+}$may be expressed in terms of one-dimensional projectors:

$$
\hat{A}=\sum_{n} \lambda_{n} \hat{P}_{n}
$$

where

$$
\hat{P}_{n}^{+}=\hat{P}_{n} ; \hat{P}_{n} \hat{P}_{m}=\delta_{n m} \hat{P}_{m} ; \sum \hat{P}_{n}=\hat{I}
$$

or, in Dirac notation:

$$
\hat{P}_{n}=\left|\varphi_{n}\right\rangle\left\langle\varphi_{n}\right|
$$

$\lambda_{n}$ are eigenvalues of operator $\hat{A}$ and $\left|\varphi_{n}\right\rangle$ are its eigenfunctions. The set of eigenfunctions forms a complete orthonormal basis. Thus, the space obtained is the metric space suitable for physical applications, hence, Operator (9) defines a pure state. More generally, one introduces the density matrix

$$
\begin{gathered}
\hat{\rho}=|\varphi\rangle\langle\varphi| \\
\rho_{i j}=\left\langle\varphi_{i}|\hat{\rho}| \varphi_{j}\right\rangle=\left\langle\varphi_{i} \mid \varphi\right\rangle\left\langle\varphi \mid \varphi_{j}\right\rangle \\
\rho_{i j}=\left\langle\varphi_{i} \mid \varphi\right\rangle\left\langle\varphi_{j} \mid \varphi\right\rangle^{*}
\end{gathered}
$$

or

$$
\hat{\rho}=\sum_{n} w_{n}\left|\varphi_{n}\right\rangle\left\langle\varphi_{n}\right|
$$

We may try to use linear algebra in order to clarify the difference between uni- and multi-component states. Using Heisenberg-Schrödinger notation, we may write:

$$
\begin{gathered}
\hat{P}_{1}=\left(\begin{array}{ll}
1 & 0 \\
0 & 0
\end{array}\right) ; \quad \hat{P}_{2}=\left(\begin{array}{ll}
0 & 0 \\
0 & 1
\end{array}\right) \\
\hat{P}_{1}+\hat{P}_{2}=\hat{I}
\end{gathered}
$$

We consider the two-component case only for its simplicity, generalization to the non-generate finite dimension case is straightforward:

Now consider the two-component wave function. Then

$$
\begin{aligned}
& |\Psi\rangle=a\left(\begin{array}{l}
1 \\
0
\end{array}\right)+b\left(\begin{array}{l}
0 \\
1
\end{array}\right)=\left(\begin{array}{l}
a \\
b
\end{array}\right) \\
& a a^{*}+b b^{*}=1
\end{aligned}
$$

The corresponding density matrix 


$$
\hat{\rho}=\left(\begin{array}{ll}
a a^{*} & a b^{*} \\
b a^{*} & b b^{*}
\end{array}\right)
$$

may be obtained using Kronecker product multiplication

$$
\hat{\rho}=\left(\begin{array}{l}
a \\
b
\end{array}\right) \otimes\left(a^{*}, b^{*}\right)
$$

However, equation (14) still describes a pure state, since

$$
\hat{\rho}=\hat{\rho}^{+} ; \hat{\rho}^{2}=\hat{\rho} ; \operatorname{Tr} \hat{\rho}=1
$$

Let us introduce the notation

$$
\hat{\tilde{\rho}}=\left(\begin{array}{cc}
a a^{*} & 0 \\
0 & 0
\end{array}\right)+\left(\begin{array}{cc}
0 & 0 \\
0 & b b^{*}
\end{array}\right)
$$

Then,

$$
\hat{\rho}=\hat{\tilde{\rho}}+\left(\begin{array}{cc}
0 & a b^{*} \\
b a^{*} & 0
\end{array}\right)
$$

Obviously,

$$
\hat{\tilde{\rho}}=\hat{\tilde{\rho}}^{+} ; \operatorname{Tr} \hat{\tilde{\rho}}=1
$$

But

$$
\hat{\tilde{\rho}}^{2} \neq \hat{\tilde{\rho}} \text { if } a b \neq 0
$$

$\hat{\tilde{\rho}}$ is a mixture of two pure one-particle states $\left(\begin{array}{l}a \\ 0\end{array}\right)$ and $\left(\begin{array}{l}0 \\ b\end{array}\right)$. Clearly, this cannot be treated as a single particle state. In order to demonstrate this let us calculate the dispersion of the projection operator $\hat{\rho}$ :

$$
\langle\hat{\rho}\rangle \equiv \operatorname{Tr}(\hat{\rho} \hat{\tilde{\rho}})=\left(a a^{*}\right)^{2}+\left(b b^{*}\right)^{2}=1-2\left(a a^{*}\right)\left(b b^{*}\right) .
$$

If $a b \neq 0$, then

$$
1>\langle\hat{\rho}\rangle>0 \text { and } 1>\langle\hat{\rho}\rangle^{2}>0
$$

Therefore,

$$
(\Delta \hat{\rho})^{2} \equiv \operatorname{Tr}\left(\hat{\rho}^{2} \hat{\tilde{\rho}}\right)-(\operatorname{Tr}(\hat{\rho} \hat{\tilde{\rho}}))^{2}=\operatorname{Tr}(\hat{\rho} \hat{\tilde{\rho}})[1-\operatorname{Tr}(\hat{\rho} \hat{\tilde{\rho}})]>0
$$

which contradicts the spectral decomposition theorem. Hence, the system state in our example is a pure state.

The operator $\hat{\rho}$ (Equation (14)) preserves the clear geometrical meaning of a one-dimensional, dispersion-free projector. If one starts with a well-defined reference frame, the complete set of those projectors allows the rotation of that new reference frame to the new axes. However, that set does not allow the extraction of information about the dispersions contained in the measurements of the transition amplitudes. The next section discusses the self-adjoint operators that allow for doing just that. 


\section{Eigenschaften Operators}

From the logical point of view, it is natural to expect that projection operators do not provide an adequate means for obtaining information about all possible alternatives, since they destroy the orthogonal subspace of the Hilbert space: A true eigenschaften operator must be unitary. Together with the requirement of being observable $\left(\hat{H}^{+}=\hat{H}\right)$, that leads to the following theorem:

Theorem:

If $\hat{H}^{+}=\hat{H}^{-1}$ (unitary) and $\hat{H}^{+}=\hat{H} \quad$ (self-adjoint),

Then

$$
\hat{H}^{2}=\hat{I} .
$$

Proof: (23)

1) Suppose

$$
\hat{H}^{+}=\hat{H}=\hat{H}^{-1},
$$

then

$$
\hat{H} \cdot \hat{H}=\hat{H} \cdot \hat{H}^{-1}=\hat{I} .
$$

2) Suppose

$$
\hat{H}^{2}=\hat{I} \text { and } \hat{H}^{+}=\hat{H}^{-1} \text {, }
$$

then

$$
\hat{H}^{+}=\hat{H} .
$$

From $\hat{H}^{2}=\hat{I}$ we have

$$
(\hat{H}-\hat{I}) \cdot(\hat{H}+\hat{I})=0
$$

Let us first consider the two-dimensional case. From Equation (24)

$$
\lambda_{1}=1 ; \lambda_{2}=-1
$$

and due to the spectral composition theorem, we have

$$
\hat{H}_{2}=\hat{P}_{1}-\hat{P}_{2}
$$

Since

$$
\hat{P}_{1}+\hat{P}_{2}=\hat{I}
$$

we finally obtain

$$
\begin{gathered}
\hat{P}_{1}=\frac{\hat{I}+\hat{H}_{2}}{2} \\
\hat{P}_{2}=\frac{\hat{I}-\hat{H}_{2}}{2}
\end{gathered}
$$

Now in terms of matrix mechanics we have

$$
\begin{gathered}
\hat{H}_{2}\left|\Psi_{1}\right\rangle=\alpha_{1}\left|\Psi_{1}\right\rangle+\beta \mathrm{e}^{i \Delta \varphi}\left|\Psi_{2}\right\rangle \equiv\left|\Psi_{3}\right\rangle \\
\hat{H}_{2}\left|\Psi_{2}\right\rangle=\beta \mathrm{e}^{-i \Delta \varphi}\left|\Psi_{1}\right\rangle+\alpha_{2}\left|\Psi_{2}\right\rangle \equiv\left|\Psi_{4}\right\rangle
\end{gathered}
$$

with 


$$
\begin{gathered}
\left\langle\Psi_{1} \mid \Psi_{1}\right\rangle=\left\langle\Psi_{2} \mid \Psi_{2}\right\rangle=\left\langle\Psi_{3} \mid \Psi_{3}\right\rangle=\left\langle\Psi_{4} \mid \Psi_{4}\right\rangle=1 \\
\left\langle\Psi_{1} \mid \Psi_{2}\right\rangle=\left\langle\Psi_{3} \mid \Psi_{4}\right\rangle=0
\end{gathered}
$$

Then,

$$
\begin{aligned}
& \beta \cdot\left(\alpha_{1}+\alpha_{2}\right)=0 \\
& \alpha_{1}^{2}+\beta^{2}=1 \\
& \alpha_{2}^{2}+\beta^{2}=1
\end{aligned}
$$

Since we are discussing here the measurement of the relevant parameters of quantum mechanical systems with non-vanishing dispersion, we consider only the $\beta \neq 0$ case. Then,

$$
\alpha_{1}=-\alpha_{2} \equiv \alpha
$$

or

$$
\operatorname{Tr}\left(\hat{H}_{2}\right)=0
$$

The matrix elements

$$
\left\langle\Psi_{1}\left|\hat{H}_{2}\right| \Psi_{1}\right\rangle=-\left\langle\Psi_{2}\left|\hat{H}_{2}\right| \Psi_{2}\right\rangle=\alpha
$$

and

$$
\left\langle\Psi_{2}\left|\hat{H}_{2}\right| \Psi_{1}\right\rangle=\beta \mathrm{e}^{i \cdot \Delta \varphi}
$$

are all we need to know about the quantum state. Both are measurable, $\left(\hat{H}_{2}\right)_{11}$ defines the spectrum and $\left(\hat{H}_{2}\right)_{12}$ defines the dispersion. The basis introduced above $\left|\Psi_{3}\right\rangle$ and $\left|\Psi_{4}\right\rangle$ is distinguished by the fact that it allows for simultaneous measurement of both spectrum and dispersion. The example of a two-level system should make this even clearer:

$$
\begin{aligned}
& \hat{H}_{2}\left(\mathrm{e}^{-i \cdot \omega_{1} t}\left|\Psi_{1}\right\rangle\right)=\alpha_{1} \mathrm{e}^{-i \cdot \omega_{1} t}\left|\Psi_{1}\right\rangle+\beta \mathrm{e}^{-i \cdot \omega_{2} t}\left|\Psi_{2}\right\rangle \\
& \hat{H}_{2}\left(\mathrm{e}^{-i \cdot \omega_{2} t}\left|\Psi_{2}\right\rangle\right)=\beta \mathrm{e}^{-i \cdot \omega_{1} t}\left|\Psi_{1}\right\rangle-\alpha \mathrm{e}^{-i \cdot \omega_{2} t}\left|\Psi_{2}\right\rangle
\end{aligned}
$$

Then dropping the overall phase factor, we obtain

$$
\begin{aligned}
& \hat{H}_{2}\left|\Psi_{1}\right\rangle=\alpha\left|\Psi_{1}\right\rangle+\beta \mathrm{e}^{i \cdot\left(\omega_{1}-\omega_{2}\right) t}\left|\Psi_{2}\right\rangle \\
& \hat{H}_{2}\left|\Psi_{2}\right\rangle=\beta \mathrm{e}^{-i \cdot\left(\omega_{1}-\omega_{2}\right) t}\left|\Psi_{1}\right\rangle-\alpha\left|\Psi_{2}\right\rangle
\end{aligned}
$$

Using the relations in (31) we obtain the most general solution:

$$
\hat{H}_{2}=\left(\begin{array}{cc}
\cos \gamma & \mathrm{e}^{-i \cdot \Delta \varphi} \cdot \sin \gamma \\
\mathrm{e}^{i \cdot \Delta \varphi} \cdot \sin \gamma & -\cos \gamma
\end{array}\right)
$$

In particular, for $\Delta \varphi=0$ and $\gamma=45^{\circ}$ we obtain the Hadamard matrix of lowest order $(N=2)$

$$
\hat{H}_{2}=\frac{1}{\sqrt{2}}\left(\begin{array}{cc}
1 & 1 \\
1 & -1
\end{array}\right)
$$

that is well-known in image processing applications.

Now we demonstrate that the solution obtained is intrinsically consistent with the general statement [3] referred to above. The density matrix in our example 
(Equation (14)) is

$$
\hat{\rho}=\left(\begin{array}{cc}
\cos ^{2} \gamma & \sin \gamma \cdot \cos \gamma \cdot \mathrm{e}^{-i \Delta \varphi} \\
\sin \gamma \cdot \cos \gamma \cdot \mathrm{e}^{i \Delta \varphi} & \sin ^{2} \gamma
\end{array}\right)
$$

Then

$$
\begin{gathered}
\left\langle\hat{H}_{2}\right\rangle \equiv \operatorname{Tr}\left(\hat{H}_{2} \hat{\rho}\right)=\operatorname{Tr}\left(\begin{array}{cc}
\cos \gamma & \sin \gamma \cdot \mathrm{e}^{-i \Delta \varphi} \\
0 & 0
\end{array}\right)=\cos \gamma=\alpha \\
\left\langle\hat{H}_{2}^{2}\right\rangle \equiv \operatorname{Tr}\left(\hat{H}_{2}^{2} \hat{\rho}\right)=\operatorname{Tr} \hat{\rho}=1
\end{gathered}
$$

and

$$
\left(\Delta \hat{H}_{2}\right)^{2} \equiv\left\langle\hat{H}_{2}^{2}\right\rangle-\left(\left\langle\hat{H}_{2}\right\rangle\right)^{2}=1-\cos ^{2} \gamma=\sin ^{2} \gamma=\beta \beta^{*}
$$

Consider now the three-component case (an analog to three-level quantum mechanical systems).

We prefer to explicitly discuss the three-component and the four-component cases, rather than the general n-dimensional situation which follows directly from the results obtained.

We have

$$
\begin{aligned}
& \hat{H}_{3}\left|\Psi_{1}\right\rangle=\alpha_{1}\left|\Psi_{1}\right\rangle+\beta e^{i \Delta \phi_{1}}\left|\Psi_{2}\right\rangle+\gamma \mathrm{e}^{i \Delta \varphi_{2}}\left|\Psi_{3}\right\rangle \equiv\left|\Psi_{4}\right\rangle \\
& \hat{H}_{3}\left|\Psi_{2}\right\rangle=\beta \mathrm{e}^{-i \Delta \varphi_{1}}\left|\Psi_{1}\right\rangle+\alpha_{2}\left|\Psi_{2}\right\rangle+\mu \mathrm{e}^{i \Delta \varphi_{3}}\left|\Psi_{3}\right\rangle \equiv\left|\Psi_{5}\right\rangle \\
& \hat{H}_{3}\left|\Psi_{3}\right\rangle=\gamma \mathrm{e}^{-i \Delta \varphi_{2}}\left|\Psi_{1}\right\rangle+\mu \mathrm{e}^{-i \Delta \varphi_{3}}\left|\Psi_{2}\right\rangle+\alpha_{3}\left|\Psi_{3}\right\rangle \equiv\left|\Psi_{6}\right\rangle
\end{aligned}
$$

with

$$
\begin{aligned}
& \left\langle\Psi_{1} \mid \Psi_{1}\right\rangle=\left\langle\Psi_{2} \mid \Psi_{2}\right\rangle=\left\langle\Psi_{3} \mid \Psi_{3}\right\rangle=1 \\
& \left\langle\Psi_{4} \mid \Psi_{4}\right\rangle=\left\langle\Psi_{5} \mid \Psi_{5}\right\rangle=\left\langle\Psi_{6} \mid \Psi_{6}\right\rangle=1 \\
& \left\langle\Psi_{1} \mid \Psi_{2}\right\rangle=\left\langle\Psi_{1} \mid \Psi_{3}\right\rangle=\left\langle\Psi_{2} \mid \Psi_{3}\right\rangle=0 \\
& \left\langle\Psi_{4} \mid \Psi_{5}\right\rangle=\left\langle\Psi_{4} \mid \Psi_{6}\right\rangle=\left\langle\Psi_{5} \mid \Psi_{6}\right\rangle=0
\end{aligned}
$$

Then, the matrix elements of $\hat{H}_{3}$ are connected by the following relations:

$$
\begin{aligned}
& \left(\operatorname{Tr}\left(\hat{H}_{3}\right)\right)^{2}=\left(\alpha_{1}+\alpha_{2}+\alpha_{3}\right)^{2}=1 \\
& \operatorname{Tr}\left(\hat{H}_{3}\right)= \pm 1 \\
& \beta^{2}=\left(1 \mp \alpha_{1}\right)\left(1 \mp \alpha_{2}\right) \\
& \gamma^{2}=\left(1 \mp \alpha_{1}\right)\left(1 \mp \alpha_{3}\right) \\
& \mu^{2}=\left(1 \mp \alpha_{2}\right)\left(1 \mp \alpha_{3}\right) \\
& \Delta \varphi_{3}=\Delta \varphi_{2}-\Delta \varphi_{1}
\end{aligned}
$$

Let us establish the connection between the eigenschaften and the projection operators here. Consider the uni-dimensional projection operators

$$
\begin{aligned}
& \hat{P}_{1}=\left(\begin{array}{ccc}
1 & 0 & 0 \\
0 & 0 & 0 \\
0 & 0 & 0
\end{array}\right) ; \hat{P}_{2}=\left(\begin{array}{ccc}
0 & 0 & 0 \\
0 & 1 & 0 \\
0 & 0 & 0
\end{array}\right) ; \hat{P}_{3}=\left(\begin{array}{ccc}
0 & 0 & 0 \\
0 & 0 & 0 \\
0 & 0 & 1
\end{array}\right) ; \\
& \hat{P}_{1}+\hat{P}_{2}+\hat{P}_{3}=\hat{I}
\end{aligned}
$$


Again, the most general one-dimensional projector may be written in the form

$$
\begin{aligned}
& \hat{\rho}=\left(\begin{array}{l}
a \\
b \\
c
\end{array}\right) \otimes\left(a^{*}, b^{*}, c^{*}\right) \\
& \hat{\rho}^{+}=\hat{\rho} ; \hat{\rho}^{2}=\hat{\rho} ; \operatorname{Tr} \hat{\rho}=a a^{*}+b b^{*}+c c^{*}=1
\end{aligned}
$$

Then using spectral decomposition

$$
\begin{aligned}
& \hat{H}_{3}=\lambda_{1} \hat{P}_{1}+\lambda_{2} \hat{P}_{2}+\lambda_{3} \hat{P}_{3} \\
& \hat{H}_{3}^{2}=\hat{I}
\end{aligned}
$$

we have

$$
\begin{aligned}
& \hat{H}_{3}^{(1)}=-\hat{P}_{1}+\hat{P}_{2}+\hat{P}_{3} \\
& \hat{H}_{3}^{(2)}=\hat{P}_{1}-\hat{P}_{2}+\hat{P}_{3} \\
& \hat{H}_{3}^{(3)}=\hat{P}_{1}+\hat{P}_{2}-\hat{P}_{3}
\end{aligned}
$$

Thus, we obtain

$$
\begin{aligned}
& \hat{P}_{1}=\frac{\hat{I}-\hat{H}_{3}^{(1)}}{2} \\
& \hat{P}_{2}=\frac{\hat{I}-\hat{H}_{3}^{(2)}}{2} \\
& \hat{P}_{3}=\frac{\hat{I}-\hat{H}_{3}^{(3)}}{2}
\end{aligned}
$$

However, only two equations are linearly independent

$$
\hat{H}_{3}^{(1)}+\hat{H}_{3}^{(2)}+\hat{H}_{3}^{(3)}=\hat{I}
$$

and form the following commutative algebra

$$
\begin{aligned}
& \hat{H}_{3}^{(1)} \cdot \hat{H}_{3}^{(2)}=-\hat{H}_{3}^{(3)} \\
& {\left[\hat{H}_{3}^{(i)}, \hat{H}_{3}^{(j)}\right]=0 ; i, j=1,2,3}
\end{aligned}
$$

We conclude with a demonstration of the four-component case. The $\hat{H}_{4}$ operators $\left(\hat{H}_{4}^{+}=\hat{H}_{4}\right.$ and $\left.\hat{H}_{4}^{2}=\hat{I}\right)$ have the form:

$$
\hat{H}_{4}=\left(\begin{array}{cccc}
\alpha_{1} & \beta e^{-i \Delta \varphi_{1}} & \gamma \mathrm{e}^{-i \Delta \varphi_{2}} & \delta e^{-i \Delta \varphi_{4}} \\
\beta \mathrm{e}^{i \Delta \varphi_{1}} & \alpha_{2} & \mu \mathrm{e}^{-i \Delta \varphi_{3}} & v \mathrm{e}^{-i \Delta \varphi_{5}} \\
\gamma \mathrm{e}^{i \Delta \varphi_{2}} & \mu \mathrm{e}^{i \Delta \varphi_{3}} & \alpha_{3} & \zeta \mathrm{e}^{-i \Delta \varphi_{6}} \\
\delta \mathrm{e}^{i \Delta \varphi_{4}} & v \mathrm{e}^{i \Delta \varphi_{5}} & \zeta \mathrm{e}^{i \Delta \varphi_{6}} & \alpha_{4}
\end{array}\right)
$$

Now we have

$$
\operatorname{Tr}\left(\hat{H}_{4}\right)=-2,0,2
$$


If $\operatorname{Tr}\left(\hat{H}_{4}\right)= \pm 2$, the transition amplitudes (dispersions) are related to the spectrum through the following equations:

$$
\begin{aligned}
& \beta^{2}=\left(1 \mp \alpha_{1}\right)\left(1 \mp \alpha_{2}\right) \\
& \gamma^{2}=\left(1 \mp \alpha_{1}\right)\left(1 \mp \alpha_{3}\right) \\
& \delta^{2}=\left(1 \mp \alpha_{1}\right)\left(1 \mp \alpha_{4}\right) \\
& \mu^{2}=\left(1 \mp \alpha_{2}\right)\left(1 \mp \alpha_{3}\right) \\
& v^{2}=\left(1 \mp \alpha_{2}\right)\left(1 \mp \alpha_{4}\right) \\
& \zeta^{2}=\left(1 \mp \alpha_{3}\right)\left(1 \mp \alpha_{4}\right)
\end{aligned}
$$

Notice that these relations are universally valid and thus are subject to direct experimental verification.

As in the above, we may establish relations between eigenschaften and projection operators. For example, for $\operatorname{Tr}\left(\hat{H}_{4}\right)=2$ we obtain

$$
\begin{aligned}
& \hat{I}=\hat{P}_{1}+\hat{P}_{2}+\hat{P}_{3}+\hat{P}_{4} \\
& \hat{H}_{4}^{(1)}=-\hat{P}_{1}+\hat{P}_{2}+\hat{P}_{3}+\hat{P}_{4}=\hat{I}-2 \hat{P}_{1} \\
& \hat{H}_{4}^{(2)}=\hat{P}_{1}-\hat{P}_{2}+\hat{P}_{3}+\hat{P}_{4}=\hat{I}-2 \hat{P}_{2} \\
& \hat{H}_{4}^{(3)}=\hat{P}_{1}+\hat{P}_{2}-\hat{P}_{3}+\hat{P}_{4}=\hat{I}-2 \hat{P}_{3} \\
& \hat{H}_{4}^{(4)}=\hat{P}_{1}+\hat{P}_{2}+\hat{P}_{3}-\hat{P}_{4}=\hat{I}-2 \hat{P}_{4}
\end{aligned}
$$

Again, we have

$$
\frac{1}{2} \sum_{i=1}^{4} \hat{H}_{4}^{(i)}=\hat{I}
$$

and

$$
\begin{aligned}
& \hat{H}_{4}^{(1)} \cdot \hat{H}_{4}^{(2)}=\hat{H}_{4}^{(1)}+\hat{H}_{4}^{(2)}-\hat{H}_{4}^{(3)}-\hat{H}_{4}^{(4)} \\
& {\left[\hat{H}_{4}^{(i)}, \hat{H}_{4}^{(j)}\right]=0 ; i, j=1,2,3,4}
\end{aligned}
$$

and so on.

For the case $\operatorname{Tr}\left(\hat{H}_{4}\right)=0$, we may write

$$
\begin{aligned}
& \hat{H}_{4}^{(1)}=I \otimes \hat{H}_{2} \\
& \hat{H}_{4}^{(2)}=\hat{H}_{2} \otimes I \\
& \hat{H}_{4}^{(3)}=\hat{H}_{2} \otimes \hat{H}_{2}
\end{aligned}
$$

since

$$
\operatorname{Tr}(A \otimes B)=\operatorname{Tr} A \cdot \operatorname{Tr} B
$$

Then we have

$$
\begin{aligned}
& \hat{I}=\hat{P}_{1}+\hat{P}_{2}+\hat{P}_{3}+\hat{P}_{4} \\
& \hat{H}_{4}^{(1)}=\hat{P}_{1}-\hat{P}_{2}+\hat{P}_{3}-\hat{P}_{4} \\
& \hat{H}_{4}^{(2)}=\hat{P}_{1}+\hat{P}_{2}-\hat{P}_{3}-\hat{P}_{4} \\
& \hat{H}_{4}^{(3)}=\hat{P}_{1}-\hat{P}_{2}-\hat{P}_{3}+\hat{P}_{4}
\end{aligned}
$$

and 


$$
\begin{aligned}
& \hat{P}_{1}=\frac{1}{4}\left[\hat{I}+\hat{H}_{4}^{(1)}+\hat{H}_{4}^{(2)}+\hat{H}_{4}^{(3)}\right] \\
& \hat{P}_{2}=\frac{1}{4}\left[\hat{I}-\hat{H}_{4}^{(1)}+\hat{H}_{4}^{(2)}-\hat{H}_{4}^{(3)}\right] \\
& \hat{P}_{3}=\frac{1}{4}\left[\hat{I}+\hat{H}_{4}^{(1)}-\hat{H}_{4}^{(2)}-\hat{H}_{4}^{(3)}\right] \\
& \hat{P}_{4}=\frac{1}{4}\left[\hat{I}-\hat{H}_{4}^{(1)}-\hat{H}_{4}^{(2)}+\hat{H}_{4}^{(3)}\right]
\end{aligned}
$$

Again we have

$$
\begin{aligned}
& \hat{H}_{4}^{(1)} \cdot \hat{H}_{4}^{(2)}=\hat{H}_{4}^{(3)} \\
& {\left[\hat{H}_{4}^{(i)}, \hat{H}_{4}^{(j)}\right]=0 ; i, j=1,2,3}
\end{aligned}
$$

We assume that the way to further generalization is obvious.

\section{Holographic Detection: Quantum Reference Frames}

Perhaps nobody needs an explanation of the mathematical formalism discussed in the previous section: we hope it speaks for itself. Nevertheless, we devote this section to the description of the physical "picture" behind the approach presented since that was the guideline that led us to it.

We address the following questions:

1) What is the difference between "on-off" and "or-and" switches in terms of quantum mechanical self-adjoint operators (observables)?

2) How are transition amplitudes between stationary (pure) states naturally and symmetrically incorporated within the amplitudes of these states?

3) Is it possible to measure $\overline{\hat{A}}$ and $\Delta \hat{A}$ simultaneously and how is the required setup arranged?

4) If it is possible, may measurements be performed using only macroscopic devices?

5) What does the Heisenberg Dispersion Relation (HDR) have to do with those measurements?

Our answer to the last question: almost nothing. It is well known [5] that the product of two noncommuting self-adjoint operators is not a self-adjoint operator and that the dispersion of their product is also not a self-adjoint operator. Therefore, there is no way to assign physical meaning to its numerical value. The theoretical importance of HDR tells us that quantum physics is the physics of extended objects and not the physics of material Newtonian points. The results of measurements are "pictures" and cannot in principle be treated as an image of a single point in space-time. The projection operators extensively used by J. von Neumann in his attempt to formulate his theory of measurements obviously play therole of "on-off" switches that define the basis of state vectors in Hilbert space. Therefore, it is reasonable to expect that "or-and" operators should be connected to them but in a slightly different way. Hadamard transformations [3] [6] which find their applications in image processing and quantum information theory seem to be suitable candidates. In addition, the notions of bits and qubitsnaturally 
appear as two-component wave packets. Finally, in order to provide a laboratory realization of the simultaneous measurement of the relevant amplitudes (relative generalized coordinates) and phase differences one should assure that wave packets arrive at every point on the detector screen.

Let us expose the content of our discussion to the eyes of the Schrödinger cat totally confused by the endless debates about its destiny. The usual justification for the apparent uncertainty refers to HDR. But empirical evidence tells us that the initial assumption that the cat may be considered as a quantum mechanical system containing inherent indeterminacy which then "becomes transformed into macroscopic indeterminacy" [7] is clearly wrong. If the state of the system (the "cat") is defined, one can measure its dispersion. Now, if in that given state the dispersion is not zero, we are dealing with an extended object and the expected result of the measurement should be represented by a picture of an unfortunate cat "mixed or smeared out in equal parts" [7]; if not, the cat was and will remain in the pure (definite) state, hopefully alive! Now, let us remember that in classical physics where only measurements of amplitudes are required, nobody doubts that the "moon is there" and that it is the same for all inertial reference frames, for example (Figure 1).

Here the lossless beam splitter is the macroscopic device which actively participates in the detection procedure $\left(\hat{H}_{2}^{+}=\hat{H}_{2}\right)$.

By contrast, in the microscopic quantum mechanical world (quantum optics) we are also required to measure the phase differences in order to obtain all existent and necessary information about the original object. This may be done using a similar setup, for example, see Figure 2.

However, in both cases the mirror and the lossless beam splitter participate only passively in the detection; they do not cause the wave function to collapse, but allow for extracting information on phase differences, since the referential component of the wave packet arrives a teach point of the detector screen
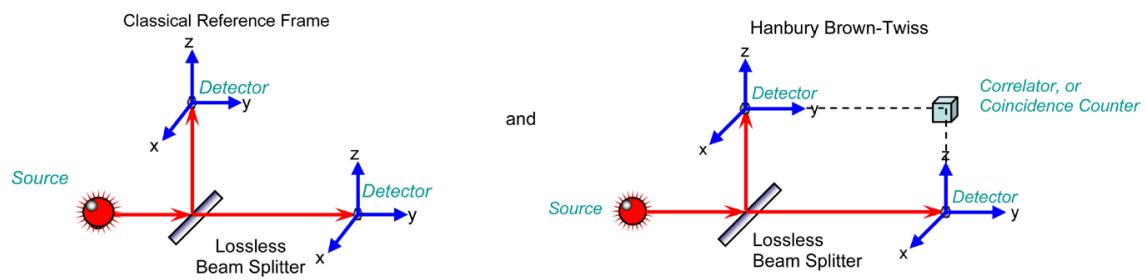

Figure 1. Classical optics measurement systems.
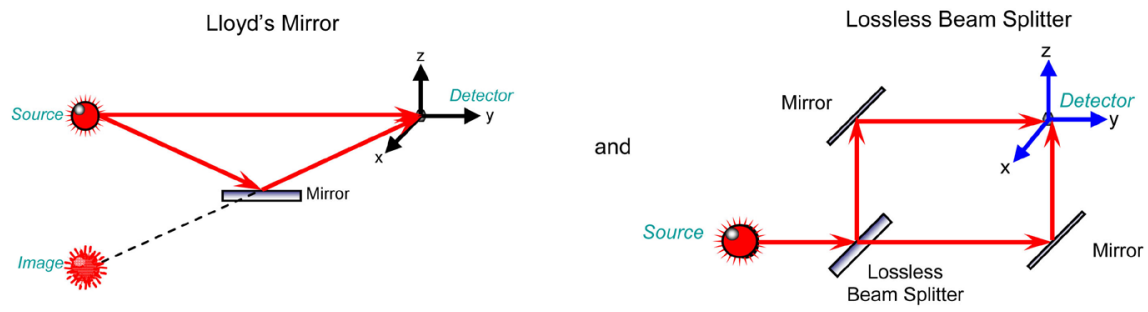

Figure 2. Quantum optics measurement systems. 
together with the tested wave packet (within the inherent dispersion of the quantum mechanical space-time continuum). Then there is no reason to expect that the picture obtained would not provide an adequate image of the original object. It seems that now we are better equipped to formulate the dynamic (relativistic) laws of quantum physics. Ultimately that should lead to deeper understanding of the geometry of the space-time continuum.

\section{Conflicts of Interest}

The author declares no conflicts of interest regarding the publication of this paper.

\section{References}

[1] von Neumann, J. (1931) MathematischeGrundlagen der Quantenmechanik. Springer, Berlin.

[2] Dirac, P.A.M. (1958) The Principles of Quantum Mechanics. 4th Edition, Clarenton Press, Oxford.

[3] Aharonov, Y. and Vaidman, L. (1990) Physical Review A, 41, 11. https://doi.org/10.1103/PhysRevA.41.11

[4] Courant, R. and Hilbert, D. (1931) Methoden der Mathematischen Physik. Springer, Berlin. https://doi.org/10.1007/978-3-642-47436-1

[5] Carruthers, P. and Nieto, M. (1968) Reviews of Modern Physics, 40, 411. https://doi.org/10.1103/RevModPhys.40.411

[6] Bouwmeester, D., Ekker, A. and Zeilinger, A. (2000) The Physics of Quantum Information.

[7] Schrödinger, E. (1935) Die Naturwissenschaften, 48, 807; 48, 823; 48, 844. 